Laser Chem. 1988, Vol. 9, pp. 123-146

(C) 1988 Harwood Academic Publishers GmbH

Photocopying permitted by license only

Reprints available directly from the Publisher

Printed in the United Kingdom

\title{
Infrared Induced Conformer Interconversion Processes in Low-Temperature Matrices
}

\author{
MARKKU RÄSÄNEN, HENRIK KUNTTU and JUHANI MURTO \\ Department of Physical Chemistry, University of Helsinki, Meritullinkatu, \\ 1-C, SF-00170 Helsinki, Finland
}

(Received January 20, 1988)

\begin{abstract}
Molecules embedded in low-temperature matrices experience conformer interconversion processes due to IR- (or UV-)irradiation. This paper summarizes the known IR-induced conformational changes, of which the majority has been studied by using broad-band sources provided with filters. We focus at the thermal and IR-photochemical methods in obtaining information of conformational energetics of matrix isolated molecules, at the usefulness of ab initio calculations in assigning the spectra of conformers involved and in finding the reaction path on the ground state surface. The data obtained from a number of molecules is used to discuss possible mechanisms in both intramolecularly hydrogen-bonded and non-bonded molecules. Also possible indications of mode-selective phenomena in these processes are dealt with. In addition, common trends found for photorotamerization in different hosts are discussed.
\end{abstract}

KEY WORDS: Conformer interconversion; ir-induced processes; low-temperature matrices.

\section{INTRODUCTION}

Since the discovery of infrared induced conformer interconversion on HONO by Pimentel and coworkers ${ }^{1,2}$ a number of papers on the topic has appeared. For $\mathrm{HONO}$ isolated in solid $\mathrm{N}_{2}$ at $20 \mathrm{~K}$ it was found that vibrational fundamental excitation was able to induce both cis to trans and trans to cis interconversion. ${ }^{2}$ The processes occur at different rates after excitation of the $\mathrm{OH}$ stretching modes, the efficiency to isomeri- 
zation by exciting the $v \mathrm{OH}($ cis $)$ being more than two orders of magnitude larger than that for the trans conformer, ${ }^{3}$ even though the molecule is brought to very similar energies by both excitations. This difference was interpreted to be due to stronger coupling between the $\mathrm{OH}$ stretching mode ${ }^{4}$ and torsion for the cis conformer than for the trans.

Expansion of studies of conformer interconversion processes started in the late 70's, when new processes on larger molecules were reported, e.g., on 2-haloethanols ${ }^{5,6}$ and on ethylene glycol. ${ }^{7}$ Most of the studies so far have employed broad band infrared sources, occasionally provided with interference filters, to induce the processes.

In some cases within limited spectral ranges interconversion processes have been studied using infrared lasers. Shirk et al. ${ }^{3,8}$ used a color center laser to study the processes on nitrous acid, and the processes on 2-halogenated ethanols. ${ }^{9-11}$ Knudsen and Pimentel ${ }^{12}$ used $\mathrm{CO}-, \mathrm{CO}_{2^{-}}$and F-center lasers to study the processes on 2,3difluoropropene in detail. Felder and Günthard ${ }^{13}$ also used a cw $\mathrm{CO}_{2}$-laser to induce frequency-selective interconversion on 1,2difluoroethane in $\mathrm{Kr}$ and $\mathrm{Xe}$ matrices. Using pulsed HF-laser, Pourcin and coworkers ${ }^{14}$ ware able to induce $\mathrm{Gg}^{\prime} \rightarrow \mathrm{Tt}$ interconversion on 2-fluoroethanol. The lack of easily available wide spectral range tunable infrared lasers has postponed detailed studies of conformer interconversion processes with the desired selectivity in excitation.

Usually the conformers interconverting by IR-irradiation are obtained with respect to rotation around a single $\mathrm{C}-\mathrm{C}, \mathrm{C}-\mathrm{O}, \mathrm{C}-\mathrm{S}$ or $\mathrm{C}-\mathrm{N}$ bond and a typical barrier to be overcome is of the order of $1500 \mathrm{~cm}^{-1}\left(18 \mathrm{~kJ} \mathrm{~mol}^{-1}\right)$. The torsional fundamental frequency connected with the reaction coordinate is below $200 \mathrm{~cm}^{-1}$ and the "active" fundamentals are near $3000 \mathrm{~cm}^{-1}$. For reaction, intramolecular energy transfer has to occur from high-energy states to the delocalized torsional states above the barrier to interconversion. Due to the low barriers the conformers interconvert fast at ambient temperatures (Boltzmann equilibrium) and one has to cool the sample in order to trap high-energy species. This can be achieved either by supersonic jet cooling or by using matrix isolation.

Even though there are intermolecular interactions between the host and guest molecules in a solid, matrix isolation renders it possible to study interconversion processes very conveniently. At low temperatures several conformers of molecules can be localized at potential energy minima. Using infrared spectroscopy one can easily identify the 
spectra of different conformers and follow the processes brought about either by infrared (or UV) irradiation or by increasing the temperature of the sample.

The search for the existence or non-existence of mode-selective phenomena in molecules is a great challenge. The most suitable systems for this purpose would be those possessing as low state densities as possible at the excitation energies. In this respect the IR-induced conformer interconversion processes, pumped at the vibrational fundamental energies, are promising. Also, since these processes are one-photon processes in the electronic ground state, one can employ accurate ab initio calculations in characterizing the conformers involved, in estimating their energetics, and perhaps most importantly, in suggesting a reaction path. Concomitantly, the experimental data provided by conformer interconversion studies in matrices is very useful for evaluating the performance of ab initio methods. In addition we can learn much about the molecular force fields by ab initio simulations of the experimental spectra of different conformers.

Previous summaries ${ }^{15-18}$ and excellent reviews ${ }^{4,19}$ on infrared induced rotational isomerization in low-temperature matrices have been published. These articles summarize the over 30 known molecules found to interconvert by infrared irradiation. The examples cover molecules with very different structures and point out the commonness of these processes. A theory on photoisomerization of impurity molecules in solids has been published. ${ }^{20}$

The aim of this paper is to summarize the studies on rotamerization performed in our laboratory; our group has discovered the majority of the known systems rotamerizing by infrared irradiation in matrices. These studies have been done by using thermal infrared sources only (with filters) to induce the processes and they can be considered as necessary preliminary results for more detailed studies with lasers. Our goal has been to identify the conformers involved in the photoprocess and to propose the reaction path. A number of suggestive results can be obtained by integrating data on processes of several molecules or by examining the processes in different matrices.

\section{EXPERIMENTAL}

In this chapter we describe some of the experimental details we have found to be important in applying matrix isolation to studies of infrared induced rotamerization. 
First of all, very high purity matrix gases must be used. On several occasions, especially when dealing with alcohols, small amounts of complexing impurities very effectively hinder the reaction. Nitrogen impurity in rare gas matrices in concentrations comparable to that of the substance can completely block the reaction. This phenomenon is most probably connected with the specific complex formation of the molecule with $\mathrm{N}_{2}$, demonstrated with well-defined complex absorptions. ${ }^{21}$ Incorporation of the low-frequency complex modes greatly increases the state density of the system, thus increasing the probability of non-reactive vibrational relaxation processes.

The concentration of the substance was kept as low as possible, since IR-induced interconversions seem to occur for monomers much more readily than for dimeric or polymeric species (IR-induced processes for intermolecularly $\mathrm{H}$-bonded systems are known for $\mathrm{H}_{2} \mathrm{O}$-dimers, methanol dimers ${ }^{23}$ and allyl alcohol dimers ${ }^{24}$ ).

The best samples were obtained by depositing the matrices at temperatures $4 \mathrm{~K}(\mathrm{Ne}), 18 \mathrm{~K}(\mathrm{Ar}), 25 \mathrm{~K}(\mathrm{Kr}), 32 \mathrm{~K}(\mathrm{Xe})$ and $16 \mathrm{~K}$ for $\mathrm{CO}$ and $\mathrm{N}_{2}$, measured with a silicon diode on the frame of the window. The true "kinetic" deposition temperature could be estimated on the basis of thermally induced processes on 2-fluoroethylamine ${ }^{25}$ as being ca. $10 \mathrm{~K}$ higher than that measured on the frame for depositing Xe at a rate of $10 \mathrm{mmol} \mathrm{h}^{-1}$. In thermal experiments slow heating and cooling rates were used $\left(2 \mathrm{~K} \mathrm{~min}^{-1}\right)$ in order to retain the integrity of the sample.

The light source for infrared irradiation was the thermal source of the spectrophotometer. To obtain "selective" IR-irradiation several filters with bandwidths of about $60 \mathrm{~cm}^{-1}$ were used. These allow selective irradiation only between different group-frequencies, and for some alcohols selective irradiation of the $\nu \mathrm{OH}$ band of individual conformers. The spectra were recorded using filters which did cut off regions able to promote photoprocesses.

\section{CONFORMATIONAL ENERGETICS}

Conformer changes studied in matrices are most important in obtaining quantitative information of conformational energetics of molecules. Conformer changes can be brought about in matrices in two ways: 
1. By warming the matrix (thermal processes), and

2. By IR and UV irradiation.

\section{Thermal experiments}

During freezing of the matrix the gas phase equilibrium is usually trapped, at least for conformers stabilized by barriers exceeding ca. $10 \mathrm{~kJ} \mathrm{~mol}^{-1}\left(835 \mathrm{~cm}^{-1}\right)$. When depositing substances at different Knudsen cell temperatures Günthard and coworkers ${ }^{26}$ have determined the conformer energy difference for several compounds. Also, trapping different gas phase thermal equilibria in solid neon at $4 \mathrm{~K}$ allowed estimation of the relative energies of the two conformers of 1-fluoropropane. ${ }^{27}$ Of course, these results refer to the gas phase and the matrix environment can alter the conformational energies to some extent. However, the similarity in energetic order of conformers in the gas phase and in matrices obtained by Knudsen cell experiments suggests that the influence of rare gases on the relative energies of conformers is not marked and that to a good approximation the properties of free molecules are applicable for matrix isolated molecules.

Combining results of thermal experiments on several molecule showing conformational behaviour, Barnes ${ }^{18}$ deduced a relationship between the threshold energy barrier and the temperature at which the thermal process is observed, e.g., at $20 \mathrm{~K}$ barriers higher than ca. $6 \mathrm{~kJ}$ $\mathrm{mol}^{-1}$ are needed for trapping the species. Annealing the matrix to about $35-40 \mathrm{~K}$ will lead to the loss of the less stable conformers for molecules with barriers of less than ca. $12 \mathrm{~kJ} \mathrm{~mol}^{-1}$. Even though the relationship is based on measurements in different matrices, and taking into account that different hosts may stabilize different species in different ways and influence the barriers, application of this "rule" has in many cases led to a semiquantitative understanding of the thermal processes, in agreement with ab initio calculated barriers. In order to make this "rule" more quantitative, there is a need for further thermal experiments in a variety of matrices.

\section{IR-photochemical experiments}

Rough estimates of the barrier heights can be obtained from filter studies, e.g., by studying the fundamental active in rotamerization. 
This method assumes that all absorptions with energies above the barrier of interconversion are active in promoting the reaction. The accuracy of this method is highly dependent on the irradiation source and benefits greatly from infrared lasers. ${ }^{12} \mathrm{We}$ have determined the barrier to isomerization for 2-chloroallyl alcohol (cis to gauche, cis being the lowest-energy species). ${ }^{28}$ The quantum yield ratio is equal to the photochemical steady state and it was found to depend on the irradiation wavelength region used. Extrapolation of the quantum yield ratio for the forward and reverse photoprocesses to zero on irradiation frequency scale yielded a barrier of $1300 \mathrm{~cm}^{-1}$, being in accordance with observations.

When the logarithm of the first-order rate constant of several IR-induced $\mathrm{C}-\mathrm{C}$ torsional processes was plotted against the respective $4-31 \mathrm{G}$ calculated barrier height $E$, a surprisingly good linear correlation resulted. ${ }^{29}$ This relationship proved to be of considerable value in suggesting routes in cases where several possibilities were at hand; glycolaldehyde is discussed as an example below. The exact form of the relationship is of course dependent upon experimental conditions such as irradiation intensity. Assuming proportionality between the rate constant and the quantum yield $\phi$, our relationship can be written approximately as $\ln \phi=-0.2_{6} E /\left(\mathrm{kJ} \mathrm{mol}^{-1}\right)$. This means that zero barrier height gives a quantum yield of unity.

This "gross relationship" suggests that the height of the barrier to be surmounted in the conformational change is of primary importance (cf. also Ref. 20). Deviations from linearity imply specific (or even mode selective?) effects; the linearity applies best to processes which lead away from the lowest energy (often hydrogen-bonded) species.

\section{Ab initio CALCULATIONS}

Processes of compounds with several conformers comprise a complicated puzzle, and ab initio calculations often provide significant evidence for solving it. They are an integral part of our studies, and we use the programs Gaussian 82 and Gaussian $86 .^{30}$

(i) Conformer energies and energy barriers In order to obtain reliable results, the geometries of all conformers must be completely optimized. The calculations refer to the vapour phase and thus neglect the 
matrix effects. However, since only conformational energy differences are of significance in interpreting the processes, this complication is usually not the dominating one. The agreement between experimental and calculated energies is often surprisingly good.

The level of the calculation should naturally be as good as possible. Use of the minimal STO-3G basis set should be avoided; it gives too low $\mathrm{C}-\mathrm{C}$ barriers and for alcohols much too high $\mathrm{C}-\mathrm{O}$ barriers. ${ }^{31}$ Increase of the size of the basis set does not, however, always improve the results (e.g., inclusion of polarization functions into the basis set of HONO leads to the incorrect result that cis is the lower-energy conformer ${ }^{32}$ ). In order to reveal the possible existence of basis-set dependence we at present optimize the geometries of all conformers and of most important energy maxima at 4-31G (in case of amines, at 4-31G($\left(\mathrm{N}^{*}\right)^{29}$ ) and $6-31 \mathrm{G}^{* *}$ levels, and carry out MP2/4-31G and MP2/6-31G** calculations using the respective HF geometries. When basis-set dependent artefacts have been removed, the calculated energies agree in most cases surprisingly well (within $1-2 \mathrm{~kJ} \mathrm{~mol}^{-1}$ ) with the "experimental" ones. The calculated barrier heights are compared with results of warming experiments (Barnes relation, see above), and the applicability of the gross barrier height relation is tested.

(ii) Calculation of vibrational spectra of conformers To confirm the conformational assignment, we usually calculate the vibrational spectrum (wavenumbers and potential energy distribution) for each conformer of the molecule in question, using ab initio force fields which refer to the optimized geometries. The problems with these calculations are, first, that they require very much computer memory (which means that $4-31 \mathrm{G}$ is often the largest possible basis-set), and second, that on the HF level the wavenumbers are ca. 10\% too large (and they must be reduced-usually with a constant factor of 0.9 ).

Glycoaldehyde $\left(\mathrm{CHOCH}_{2} \mathrm{OH}\right)$ serves as a good example of the usefulness of $\mathrm{ab}$ initio calculations in explaining observations. This small two-rotor compound has four different conformers, $\mathrm{Cc}, \mathrm{Ct}, \mathrm{Tg}$ and $\mathrm{Tt}$ (see Figures 1 and 2), of which the species Cc (all-cis) is the lowest-energy one. ${ }^{33}$ This conformer has a a quite strong intramolecular hydrogen bond (its $\nu \mathrm{OH}$ fundamental frequency is $130 \mathrm{~cm}^{-1}$ smaller than that of species $\mathrm{Tt}$ ). The interaction is so strong that, instead of the "natural" conformer $\mathrm{Cg}$, the forced conformer Cc exists. 

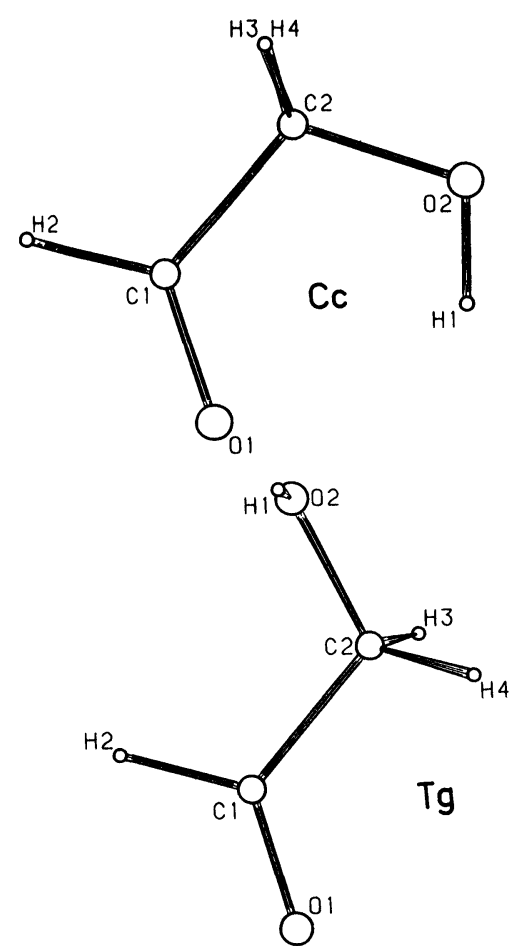
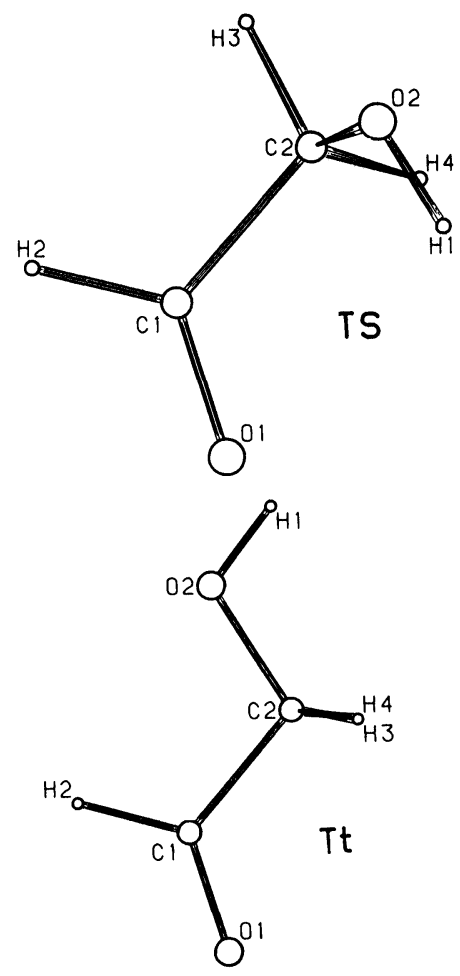

Figure 1 The conformers $\mathrm{Cc}, \mathrm{Tg}$ and $\mathrm{Tt}$ of glycolaldehyde. TS represents the transition state structure in the process $\mathrm{C} \rightarrow \mathrm{TS} \rightarrow \mathrm{Tg}, \mathrm{Tt}$.

The conformational $\mathrm{Cc} \rightarrow \mathrm{Tt}$ change is seen to occur upon IR irradiation, at $13 \mathrm{~K}$ it process to $90 \%$ completion. Instead of the usual pure $\mathrm{C}-\mathrm{C}$ rotation (vertical process in Figure 2), a combined $\mathrm{C}-\mathrm{O}$ and $\mathrm{C}-\mathrm{C}$ rotation now occurs (dotted lines: $\mathrm{TS}=$ transition state). The calculated barrier height of $20 \mathrm{~kJ} \mathrm{~mol}^{-1}$ is in agreement with the value of $19 \mathrm{~kJ} \mathrm{~mol}^{-1}$ obtained using the gross barrier height relationship and the half-life of $1.6 \mathrm{~h}$ (in Ar).

Glycolaldehyde is so far the only known molecule for which the photochemical steady state is temperature-dependent (in $\mathrm{Kr}$ and $\mathrm{Xe}$ matrices). This can be explained by assuming a thermal equilibrium between species $\mathrm{Tg}$ and $\mathrm{Tt}$ : the barrier between them is low (interconversion fast) and their energy difference is small. For the calculated $\mathrm{Tg}$-Tt energy difference of $1 \mathrm{~kJ} \mathrm{~mol}^{-1}$, the concentration ratio $\mathrm{Tg} / \mathrm{Tt}$ 


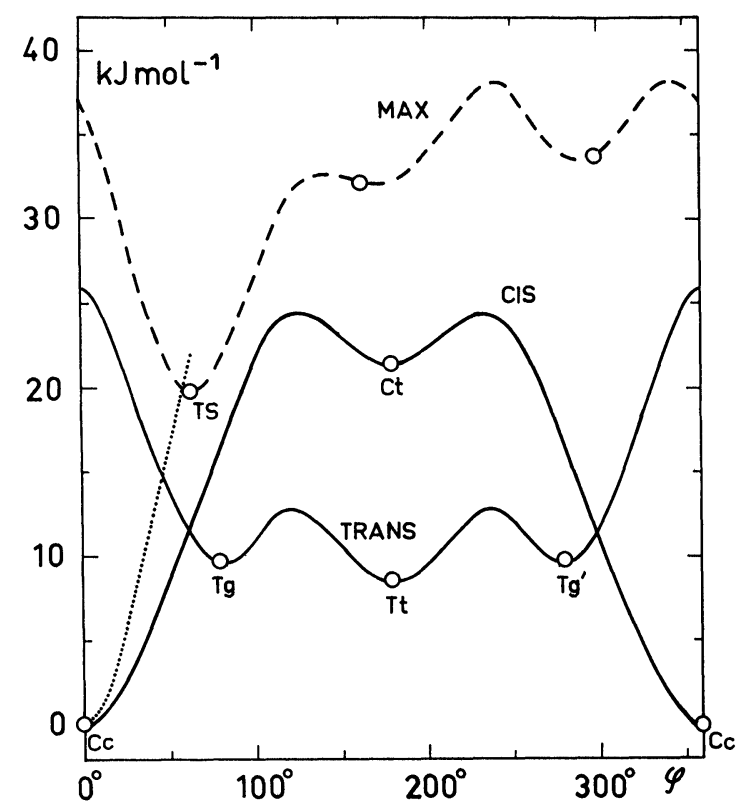

Figure 2 Torsional ab initio 6-31G** calculated potential energy curves for glycolaldehyde. Each curve refers to $\mathrm{OH}$-rotation at a constant $\mathrm{C}-\mathrm{C}$ angle. In processes between $\mathrm{C}$ and $\mathrm{T}$ conformers the broken curve has to be surmounted.

would be 0.0002 at $13 \mathrm{~K}$ and at this temperature the reverse, vertical photoprocess is then from $\mathrm{Tt}$ and is slow due to high $\mathrm{C}-\mathrm{C}$ barrier. On the other hand, at $30 \mathrm{~K}$ the concentration ratio is 0.036 , and the reverse, vertical photoprocess is from $\mathrm{Tg}$ and is fast. It accounts for the fact that the observations can be quantitatively explained in terms of the gross barrier height relationship.

One more example of the accuracy of the energetics is available in this example. The threshold temperature for the thermal process $\mathrm{Tt} \rightarrow \mathrm{Cc}$ is $30-32 \mathrm{~K}$ (in $\mathrm{Xe}$ ) for which the Barnes relation gives an effective barrier height of $9-11 \mathrm{~kJ} \mathrm{~mol}^{-1}$. The ab initio calculated barrier height for the route $\mathrm{Tt} \rightarrow \mathrm{TS} \rightarrow \mathrm{Cc}$ is $11 \mathrm{~kJ} \mathrm{~mol}^{-1}$.

\section{INTRAMOLECULARLY H-BONDED SYSTEMS}

In a great number of known IR-interconverting molecules one of the conformers has an intramolecular $\mathrm{H}$-bond and this particular conformer is usually the lowest-energy one. IR-induced interconversion 


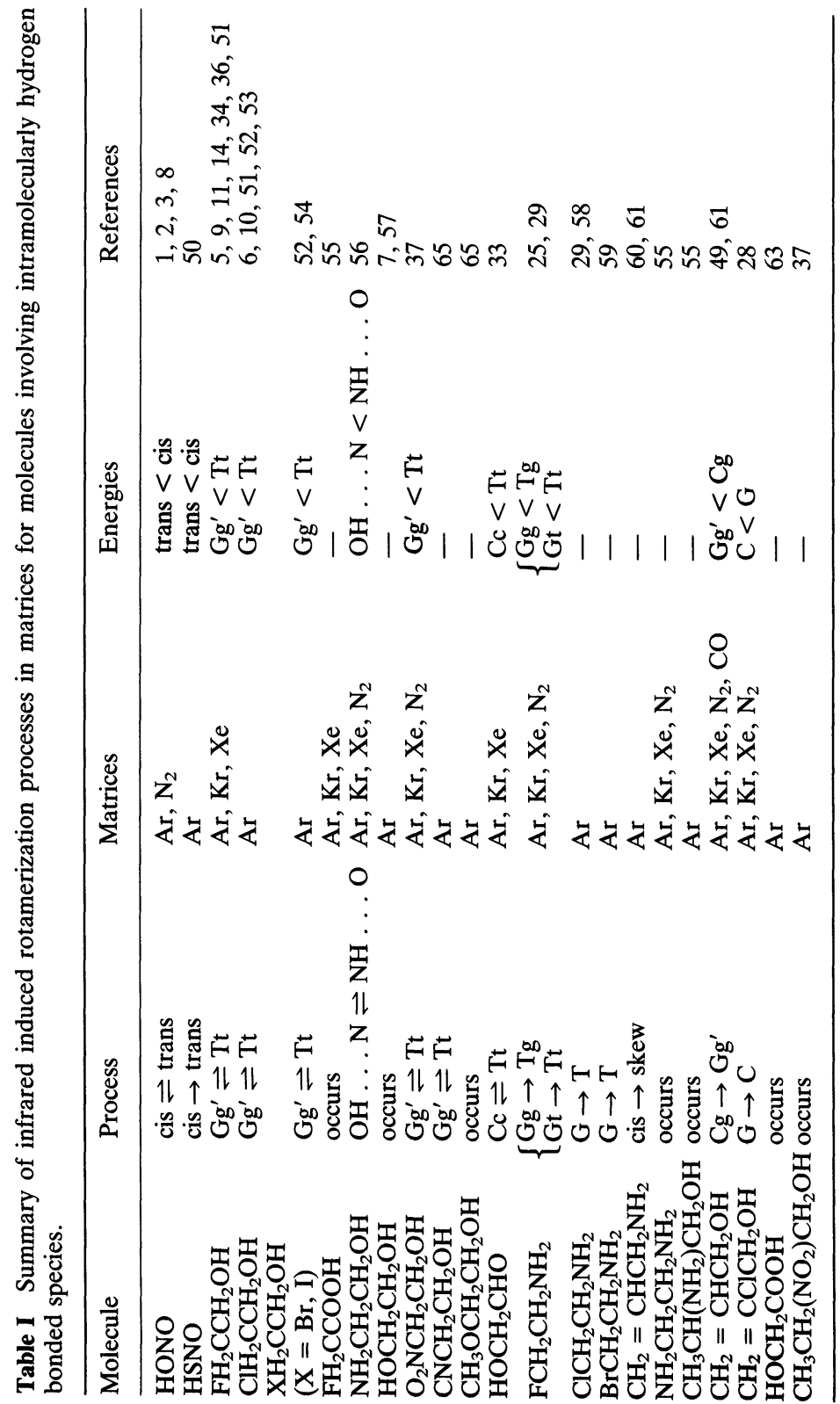


seems to favour routes away from the $\mathrm{H}$-bonded species. The exact role of the intramolecular interaction in the mechanism is not known, however it is possible that this interaction provides mechanisms for effective transfer of energy from the high-energy fundamental levels to the highly excited delocalized torsional modes. The known IRinterconverting systems with possible $\mathrm{H}$-bonded species are collected in Table I.

The most extensively studied such cases are the 2-substituted ethanols. For these IR-conversion occurs provided the barriers between conformers can be overcome at the $\mathrm{OH}$-stretching fundamental energies. ${ }^{31}$ No interconversions between OH-conformers in matrices have been observed so far and the reactant and product species are determined by the barriers with respect to rotation about the central $\mathrm{C}-\mathrm{C}$ bond. ${ }^{34}$

The most studied of 2-substituted ethanols, 2-fluorethanol (2-FE) has five different conformers $\mathrm{Gg}^{\prime}, \mathrm{Tt}, \mathrm{Tg}, \mathrm{Gt}$ and $\mathrm{Gg}$ (the capital letter refers to the dihedral angle $\mathrm{O}-\mathrm{C}-\mathrm{C}-\mathrm{F}$, the lower-case letter to the dihedral angle $\mathrm{H}-\mathrm{O}-\mathrm{C}-\mathrm{C}) .{ }^{35}$ The ab initio calculations suggest that only species $\mathrm{Gg}^{\prime}$ and $\mathrm{Tt}$ are stable at the matrix temperatures, in accordance with observations. ${ }^{34,36}$ The initial deposit of 2-FE consists of $91 \% \mathrm{Gg}^{\prime}$ and $9 \% \mathrm{Tt}$, indicating that $\mathrm{Tt}$ is $\sim 300 \mathrm{~cm}^{-1}$ higher in energy. Under broad band infrared irradiation a photochemical steady state with $14 \% \mathrm{Gg}^{\prime}$ and $86 \% \mathrm{Tt}$ is obtained in Ne at $4 \mathrm{~K} \cdot{ }^{34}$ Both $v \mathrm{CH}$ and $\nu \mathrm{OH}(\mathrm{OD})$ are active in promoting the same interconversion. Simple explanations for the preference of this uphill photoprocess are excluded since the $v \mathrm{OH}$ and $v \mathrm{CH}$ intensities are very similar for both conformers. The implications of intramolecular $\mathrm{H}$-bonding in the mechanism of conformer interconversion have been discussed by Frei and Pimentel. ${ }^{4}$

2-Nitroethanol (2-NE) represents 2-substituted ethanols where interconversion has been studied in a variety of matrices. ${ }^{37}$ Although 2-NE is a three-rotor system, it can be approximated as a two-rotor case-rotation of the nitro-group is either free or thre is only one maximum during a $180^{\circ}$ rotation and hence the nitro-group does not give rise to new conformers. ${ }^{37}$ In matrices the only stable conformers are $\mathrm{Gg}^{\prime}$ (the lowest-energy one) and $\mathrm{Tt}$, their energy difference being about $19 \mathrm{~kJ} \mathrm{~mol}^{-1}$. Intramolecular $\mathrm{H}$-bond in conformer $\mathrm{Gg}^{\prime}$ between the $\mathrm{OH}$ - and $\mathrm{NO}_{2}$-groups is demonstrated by the $45 \mathrm{~cm}^{-1} v \mathrm{OH}$ frequency difference between the conformers. 


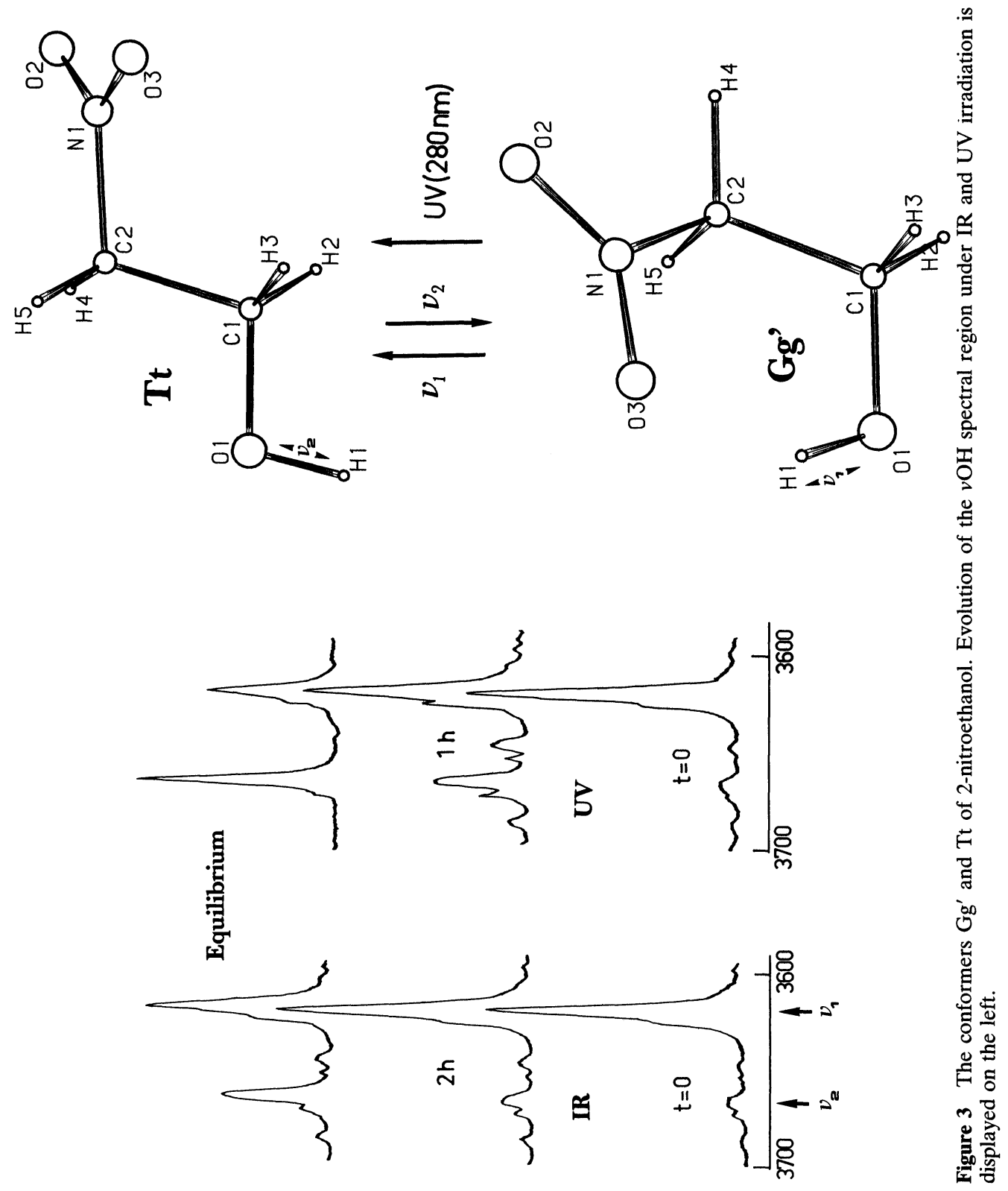


Conformer $\mathrm{Gg}^{\prime}$ is transformed into the higher-energy $\mathrm{Tt}$ conformer by irradiating at its $\mathrm{OH}$ stretching frequency $v_{1}$ and the photoprocess is reversible: irradiating at the $\mathrm{OH}$-stretching frequency of $\mathrm{Tt} v_{2}$ induces the reverse of the process. Figure 3 displays the two conformers of 2-NE, the process and the spectral evolution of the $v \mathrm{OH}$ region in solid $\mathrm{Ar}$ at $13 \mathrm{~K}$. The same interconversion on 2-NE occurs by UV-excitation at wavelengths of about $280 \mathrm{~nm}$. Light-induced processes on 2-NE do not proceed to completion, a photochemical equilibrium between $\mathrm{Gg}^{\prime}$ and $\mathrm{Tt}$ is obtained. Broad band IR-irradiation leds to $\mathrm{Tt} / \mathrm{Gg}^{\prime}$ ratio of 0.6 and UV irradiation to almost equal abundancies for both conformers. Similar equilibration of conformers due to UV irradiation has been found for various formic acid esters. ${ }^{38}$ At the present time no explanation is available for this difference in photochemical equilibrium at different wavelengths- the mechanism for interconversion after electronic excitation may differ much from that after vibrational excitation.

Figure 4 displays the potential energy surface for 2-NE, calculated at the 3-21G basis set level. The forward photoprocess occurs along the dotted line from the $\sim 60^{\circ}$ curve minimum $\left(\mathrm{Gg}^{\prime}\right)$ over the $120^{\circ}$ curve to the minimum $\mathrm{Tt}$ on the $\sim 180^{\circ}$ curve (the figures refer to the dihedral angle $\mathrm{O}-\mathrm{C}-\mathrm{C}-\mathrm{N}$ ). With reference to Figure 4 one can note that insertion of one $v \mathrm{OH}$ quantum to conformer $\mathrm{Gg}^{\prime}$ brings the molecule slightly above the barrier to interconversion, absorption of one $v \mathrm{OH}$ ( $\mathrm{Tt}$ ) photon provides the molecule with about $5000 \mathrm{~cm}^{-1}$ energy, well above the barrier to interconversion. It is striking that the uphill photoprocess still prevails under broad band IR irradiation.

Another aspect is introduced while comparing the photoprocess of 2-NE with that of 2-FE. The heavy nitro-group definitely increases the state density of 2-NE from that of 2-FE. This suggests that statistical excitation of the reaction coordinate at energies above the barrier would become more improbable. However, observation of interconversion for 2-NE supports the non-statistical nature of interconversion processes. Similar conclusions were made with fluorinated hydrocarbons, where IR-induced interconversion could easily be detected for 1-fluorononane, even though its intrinsic state density is high. ${ }^{47}$

\section{NON-H-BONDED MOLECULES}

Table II collects the known IR-interconverting molecules where no 


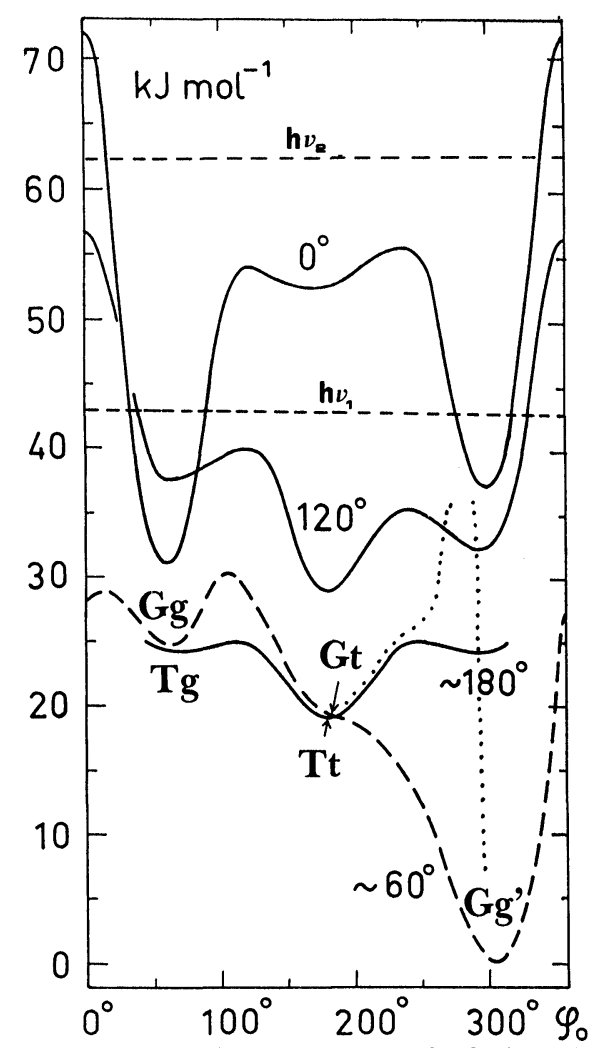

Figure 4 Semi-rigid 3-21G potential energy curves for 2-nitroethanol. The energies of the molecule after absorption of a $v \mathrm{OH}$ quantum is indicated by horizontal broken lines.

intramolecular interactions are present. The large number of such systems pinpoints the generality of rotamerization and several simple two-conformer systems can be found in Table II. With molecules possessing only two conformers identification of the species in matrices is not difficult. All of the 1-halogenated propanes have two conformers, gauche and trans. For chloro-, bromo- and iodopropane the trans form is slightly more stable than the gauche in the gas phase as verified by freezing the gas phase equilibrium in solid neon. ${ }^{27}$

For 1-fluoropropane (1-FP) the gauche is the lower-energy species in the gas phase. ${ }^{27,39}$ The potential energy surface for 1-FP with respect to rotation about the central $\mathrm{C}-\mathrm{C}$ axis is represented by a three-fold curve. With respect to IR-rotamerization the barrier heights and 


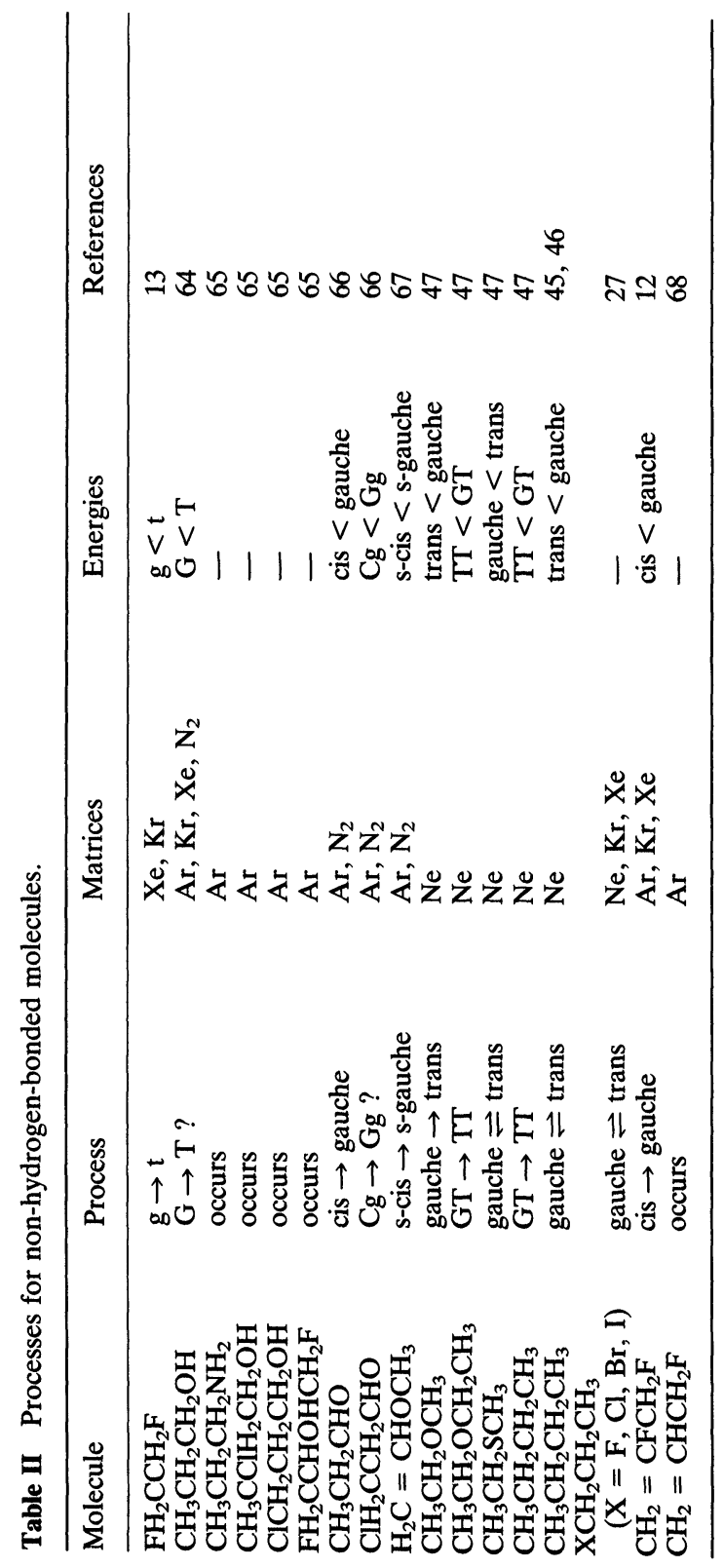


conformer energies are of interest. The experimental gas phase conformer energy difference is $170 \mathrm{~cm}^{-1},{ }^{39}$ or $130 \mathrm{~cm}^{-1},{ }^{40}$ being in agreement with the ab initio MP2/4-31G/HF/4-31G calculated value of $126 \mathrm{~cm}^{-1}{ }^{41}$ The experimental gauche $\rightarrow$ trans barrier is $1455 \mathrm{~cm}^{-1}$, 39 or $1284 \mathrm{~cm}^{-1},{ }^{40}$ and the gauche $\leftrightarrow$ gauche barrier $1524 \mathrm{~cm}^{-1} .{ }^{39}$ The calculated barrier heights depend only slightly on the level of the calculation, being on the above-mentioned level $1597 \mathrm{~cm}^{-1}(\mathrm{~g} \rightarrow \mathrm{t})$ and 1513 $\mathrm{cm}^{-1}(\mathrm{~g} \leftrightarrow \mathrm{g})$. Slight differences into these figures can be introduced while embedding the conformers into solid rare gas matrices. However, absorption of a $v \mathrm{CH}$ quantum raises the molecule to energies well above the barriers to internal rotation about the $\mathrm{C}-\mathrm{C}$-bond.

Experimentally it was found that for all of the 1-halopropanes the $v \mathrm{CH}$ region is active in promoting interconversion. For 1-FP and 1-chloropropane the process occurs readily in all rare gases, with the exception of 1-chloropropane in Ar. Similarly anomaly in the reaction rates in Ar has been found for 1,2-difluoroethane. ${ }^{13}$ The $\mathrm{C}-\mathrm{C}$ barrier heights for interconversion for these molecules do not differ drastically from those for 1-FP ${ }^{42-44}$ and this sudden inhibition of the photoprocesses in $\mathrm{Ar}$ is puzzling. If the $\nu \mathrm{CH}$ quantum were directly transferred to the torsional states, inhibition of the reaction would require a barrier height increase of about $1000-1500 \mathrm{~cm}^{-1}$ due to the Ar host. A possible explanation for this will be discussed later.

Under broad-band IR-irradiation a photochemical steady state is obtained for all of the 1-halopropanes. The process from gauche to trans prevails by about a factor of ten over the reverse photoprocess. This tendency for gauche $\rightarrow$ trans processes is not limited to halopropanes but it has been found for several other simple two-conformer systems under the influence of broad band infrared irradiation. For 1,2-difluoroethane the uphill process gauche $\rightarrow$ trans dominates, ${ }^{13}$ similar trend has been found for $n$-butane ${ }^{45}$ and its deuterated counterparts, ${ }^{46}$ as well as for ethylmethyl ether and ethylmethyl sulphide. ${ }^{47}$ The differences between $v \mathrm{CH}$ absorption intensities of different conformers are small and cannot account for this phenomenon. The observed photoprocess occurs against what one would expect according to statistical energy distribution in the highly excited molecule. Being in the free internal rotation region with respect to the reaction coordinate the molecule will "see" roughly twice as much of the surface representing the gauche conformation as the trans conformation. Why the molecule likes to relax into the trans conformation is 
unclear. One explanation suggests ${ }^{45}$ that this may be connected with the Fermi-resonances between $v \mathrm{CH}$ levels and the overtone levels of $\mathrm{CH}$ bendings. Energy dissipation would first proceed rapidly due to this type of resonance to the $\mathrm{CH}$ bending levels, and in turn to overtones of the torsion. The lower symmetry would allow more Fermi-resonances for the gauche conformer than for the trans, thus favouring processes from the lower to the higher symmetry species.

In fact this mechanism could partly explain also the non-observability of the photo-processes in $\mathrm{Ar}$, discussed above. The $\mathrm{CH}$ bending levels are very close to the barrier heights for interconversion. If these levels are critical in the energy transfer processes then slight increase of the barrier height due to the matrix can effectively block the reaction.

Dealing with the 1-halopropanes, a few things deserve attention. The rotamerization rates for different propanes decrease with increasing mass of the halogen substituent, being fastest for 1-FP, slower by a factor of two for 1-chloropropane, and slowing down by another factor of two for the heavier substituents. The influence of the mass of the substituent is less than one would expect at a first glance. Apparently one has to view the process as a motion and rearrangement of the propyl-group, with the rotamerization requiring little motion of the heavier halogen substituents.

$n$-Butane is another two-conformer system, where IR-induced conformer interconversion occurs, ${ }^{45}$ the $v \mathrm{CH}$-region being the main channel in initiating the photoprocesses. The quantum yield for the gauche $\rightarrow$ trans is about eight times higher than that for the reverse process. The gauche $\rightarrow$ trans barrier is about $1000 \mathrm{~cm}^{-1}$ and the reverse barrier $1250 \mathrm{~cm}^{-1}$. The gauche $\leftrightarrow$ gauche barrier is ca. $2000 \mathrm{~cm}^{-1}$. Absorption of one $v \mathrm{CH}$ quantum brings the molecule above all of the internal rotational barriers connected with rotation about the central $\mathrm{C}-\mathrm{C}$ bond. Figure 5 displays the energetics encountered in the photoprocesses on $n$-butane.

Since no selective IR-source was available for irradiating the different $\nu \mathrm{CH}$ absorptions, another approach was chosen: different deuterated $n$-butanes were studied under the influence of broad band IRirradiation. ${ }^{46}$

First of all, the fully deuterated species $\mathrm{C}_{4} \mathrm{D}_{10}$ did not interconvert in $\mathrm{Ne}$ at all. This is surprising, since the $v \mathrm{CD}$ energies are well above the barriers to interconversion. Based on this observation we now assume that for some reason the $v \mathrm{CD}$ absorptions are inactive in promoting 


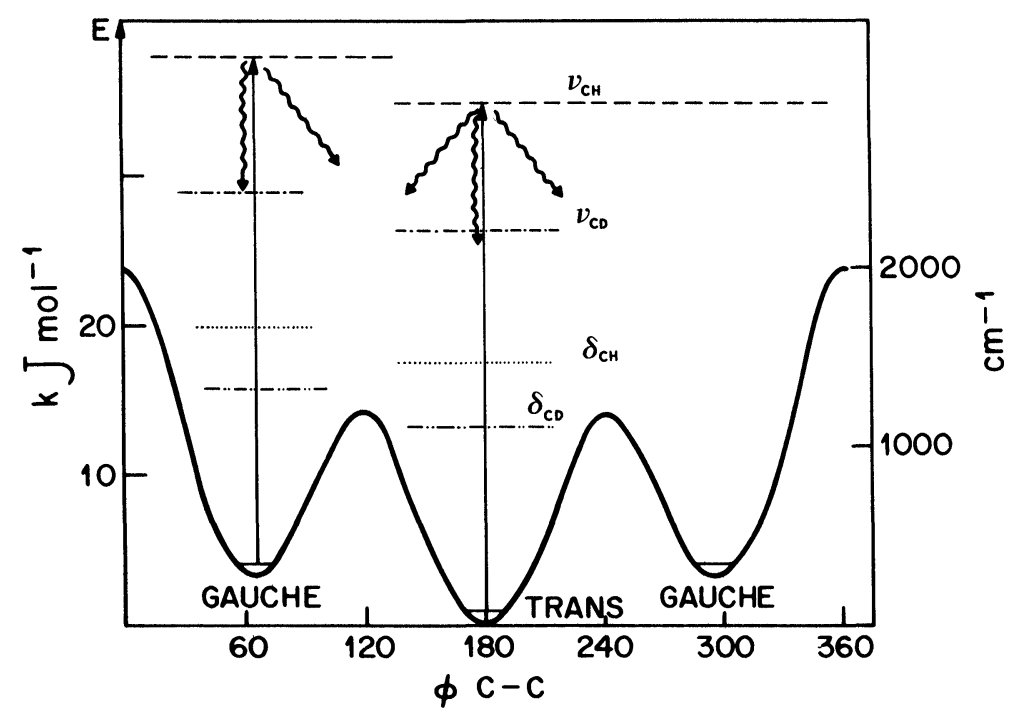

Figure $5 \mathrm{C}-\mathrm{C}$ torsional potential for $n$-butane. The energies of $\mathrm{CH}(\mathrm{CD})$ stretching and bending modes are indicated by horizontal lines.

the reaction. In a purely statistical model the reaction rate for different deuterated $n$-butanes should depend linearly on the $\mathrm{CH}$-stretching absorption intensity. For deuterated $n$-butanes it was found that the $\mathrm{CH}$-stretching intensity is linearly dependent on the number of $\mathrm{CH}$ bonds in the molecule. The primary result of this simple experiment is displayed in Figure 6a, equal amounts of each compound were isolated in Ne and irradiated with the same source. The $\mathrm{CD}_{3} \mathrm{CH}_{2} \mathrm{CH}_{2} \mathrm{CD}_{3}\left(\mathrm{~d}_{6}\right)$ compound behaves like the fully deuterated $\left(\mathrm{d}_{10}\right)$ butane, and the rotamerization is only marginally observable. On the other hand, the $\mathrm{CH}_{3} \mathrm{CD}_{2} \mathrm{CD}_{2} \mathrm{CD}_{3}\left(\mathrm{~d}_{7}\right)$ butane reacts quite readily, with the initial rate being at least a factor of 5 larger than that for $d_{6}$. This seems to indicate that the terminal $\mathrm{CH}_{3}$ groups are much more effective at promoting the rotamerization than excitation of the methylenes. The situation is somewhat more complex, as can be seen by comparing the rates in the $d_{7}, d_{5}$ and $d_{3}$ compounds. The presence of the methylene group in the $\mathrm{CH}_{3} \mathrm{CH}_{2} \mathrm{CD}_{2} \mathrm{CD}_{3}$ compound enhances the rate by an additional factor of ca. 2.5, compared with the $d_{7}$ compound. On the other hand, introduction of additional $\mathrm{CH}_{2}$-group in $\mathrm{d}_{3}$-butane actually slows down the rotamerization somewhat. This non-linearity of the rotamerization rate upon integrated $\nu \mathrm{CH}$ absorption intensity for different deuterated 

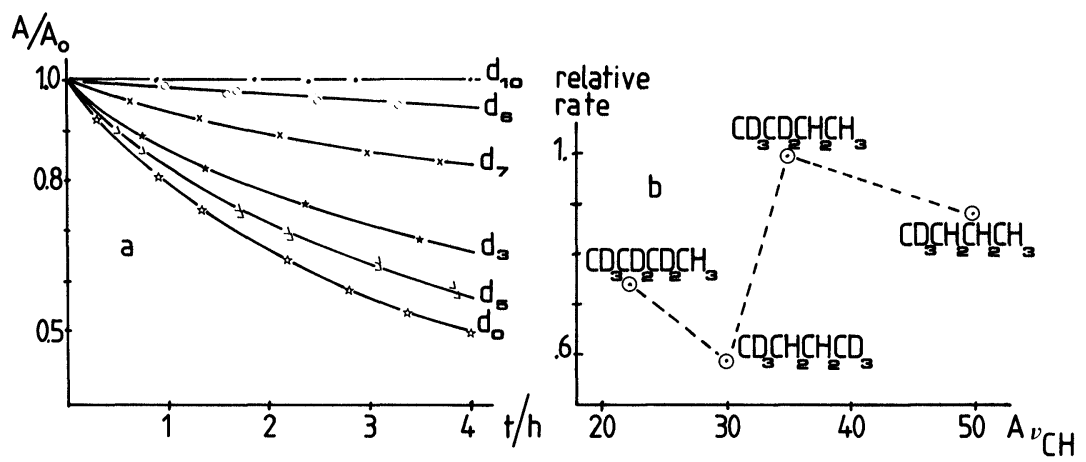

Figure 6 (a) The rates of decrease of gauche absorptions of different deuterated $n$-butanes in solid Ne under broad band IR-irradiation. (b) The relative isomerization rates for partially deuterated $n$-butanes with respect to integrated $\mathrm{CH}$-absorption intensity.

$n$-butanes is emphasized in Figure $6 \mathrm{~b}$. These results point to the importance of which modes are excited and of the details of intermode coupling, rather than to a purely random, statistical process.

The mechanism by which the energy is channeled into the reaction coordinate, delocalized torsional states, is not quite clear. Interestingly, the $\mathrm{CH}$ bending frequencies are above, while those of $\mathrm{CD}$ are $\sim$ below the rotamerization barrier. It is possible that Fermiresonances between $\nu \mathrm{CH}(\mathrm{CD})$ and $2 \times \delta \mathrm{CH}(\mathrm{CD})$-levels play a central role in this case also, and the data indicate that the mechanism does not involve an unrestricted, statistical IVR. If energy redistribution were to occur prior to reaction, it should make little difference whether the methyl or methylene $\mathrm{CH}$ stretches are the absorbers.

\section{PROCESSES IN DIFFERENT HOSTS}

The rotamerization rates in different hosts are fairly different, interconversion rates in a variety of matrices on studied molecules are collected in Table III.

Of rare gases, neon is the least perturbing - the vibrational frequencies for molecules isolated in a neon are quite close to the gas phase values. Also, no serious site-effects have been found in neon matrices. In other rare gases, however, site-effects often are serious. In Xe, for instance, the photoprocess on 1-chloropropane occurs in the main site 
Table III Host dependence of rotamerization rates under broad band IR irradiation.

\begin{tabular}{llllll}
\hline Molecule & $\mathrm{Ar}$ & $\mathrm{Kr}$ & $\mathrm{Xe}$ & $\mathrm{N}_{2}$ & References \\
\hline $\mathrm{CH}_{2}=\mathrm{CHCH}_{2} \mathrm{OH}$ & 1 & 1.1 & $5.5^{\mathrm{a}}$ & 0.13 & 49 \\
$\mathrm{O}_{2} \mathrm{NCH}_{2} \mathrm{CH}_{2} \mathrm{OH}$ & 1 & 2.7 & $30^{\mathrm{a}}$ & 0.7 & 37 \\
$\mathrm{CH}_{3} \mathrm{CH}_{2} \mathrm{CH}_{2} \mathrm{OH}$ & 1 & 2.2 & $15^{\mathrm{a}} ; 4.7^{\mathrm{b}}$ & 0.06 & 64 \\
$\mathrm{FH}_{2} \mathrm{CCH}_{2} \mathrm{OH}$ & 1 & 1.2 & $3^{\mathrm{a}}$ & - & 15 \\
$\mathrm{FH}_{2} \mathrm{CCH}_{2} \mathrm{NH}_{2}$ & 1 & 0.8 & $6^{\mathrm{a}}, 3^{\mathrm{b}}$ & 0.1 & 29 \\
$\mathrm{FH}_{2} \mathrm{CCH}_{2} \mathrm{CH}_{3}$ & 1 & 0.8 & 1.6 & - & 27 \\
$\mathrm{ClH}_{2} \mathrm{CCH}_{2} \mathrm{CH}_{3}$ & $1^{\mathrm{c}}$ & 16 & 97 & - & 27 \\
\hline
\end{tabular}

${ }^{\text {a }}$ Dep at $15 \mathrm{~K}$.

${ }^{b}$ Dep at $25 \mathrm{~K}$.

${ }^{c}$ Anomalously slow.

at about threefold rate to that in the minor site. ${ }^{27}$ The "slow" sites often make accurate estimation of the photochemical steady-state rather difficult, especially in cases where absorption bands for species in different sites overlap seriously.

From Table III it is, however, evident that there is a trend in the reaction rates in different hosts. For several alcohols and amines the relative rates are $\mathrm{Xe} \gg \mathrm{Kr} \geq \mathrm{Ar} \gg \mathrm{N}_{2}, \mathrm{CO}$. In complexing matrices the rates are an order of magnitude slower than in rare gases, the obvious reason for this being the "supermolecule" nature of molecules (especially alcohols and amines) isolated in $\mathrm{N}_{2}$ or $\mathrm{CO}$, as discussed earlier. In neon matrices, the rates for the studied 1-halopropanes ${ }^{27}$ are similar to those in $\mathrm{Kr}$.

In Xe-matrices the rotamerization rates were found to depend upon matrix deposition temperature ${ }^{25}$ no such dependence was found in $\mathrm{Ar}$ or $\mathrm{Kr}$. In Xe the rates for samples deposited at $\sim 30 \mathrm{~K}$ are usually a factor of 3 slower than for deposits obtained at $15 \mathrm{~K}$. This dependence may be connected with the observation of Schulze and $\mathrm{Kolb}^{48}$ that the density of solid Xe depends on the deposition temperature, being high for deposits obtained at high temperature $(\sim 30 \mathrm{~K})$. From the point of the rotamerizing molecule this means that the average available volume changes with deposition temperature. Some kind of "activation volume" is connected with the interconversion and the correlation between isomerization rates and host densities seems to be qualitatively reasonable. ${ }^{25}$

Taking into account the effects due to different rates in different sites the equilibrium conformer ratio of an IR-interconverting molecule under broad band irradiation seems to be independent of the 
matrix medium. ${ }^{27,33,37,49}$ This indicates that intramolecular factors determine the ratio between the opposite reaction rates.

\section{Concluding remarks}

The preliminary work done by using broad band sources to induce conformer changes in low-temperature matrices has shown the commonness of these processes. Even though the host influences the rates of the processes relatively much, the same photochemical conformer equilibrium is reached for a molecule in all matrices. This indicates that intramolecular factors are responsible for the equilibrium, and the host influences opposite photoprocesses similarly.

The problems due to sites are most serious in the heavier rare gases, and neon is the least-perturbing medium. However, studies of thermally activated processes are very important and these can be best be performed in Xe matrices. Despite differences in the reaction rates in different sites there is a well established trend for the rates in different matrices, photoprocesses being fastest in $\mathrm{Xe}$, somewhat slower in $\mathrm{Ne}$, $\mathrm{Kr}$ and $\mathrm{Ar}$ and slower by one order of magnitude in complexing media, $\mathrm{N}_{2}$ and $\mathrm{CO}$. Most probably the influences of the solid on the impurity molecule are twofold, steric (size of the cage) and electrostatic.

Studies on rotamerization are important in unravelling conformational energetics of molecules, and simulations using ab initio methods are of great value in solving the conformers in question, in estimating their energies and possible reaction paths. Several of the observed phenomena can qualitatively be explained by using the relation between the barrier height and the temperature needed to overcome it (Barnes relation), and by using the gross barrier height relationship, relating the quantum yield and the barrier height. However, several questions remain unanswered: what is the driving force for the uphill photoprocesses and why does the molecule relax in one particular conformation? Certainly, the product ratios obtained by IR-activation of the molecules differ from those obtained by thermal activation. The available data suggests that intramolecular vibrational redistribution does not occur statistically prior to reaction but certain preferences are involved in the photorotamerization processes. Much work is still to be done using wide spectral range tunable infrared lasers with the desired selectivity in excitation for getting detailed answers to the questions encountered. 


\section{References}

1. J. D. Baldeschwieler and G. C. Pimentel, J. Chem. Phys. 33, 1008 (1960).

2. R. T. Hall and G. C. Pimentel, J. Chem. Phys. 38, 1889 (1963).

3. P. A. McDonald and J. S. Shirk, J. Chem. Phys. 77, 2355 (1982).

4. H. Frei and G. C. Pimentel, Ann. Rev. Phys. Chem. 36, 491 (1985).

5. M. Perttilä, J. Murto, A. Kivinen and K. Turunen, Spectrochim. Acta 34A, 9 (1978).

6. M. Perttilä, J. Murto and L. Halonen, Spectrochim Acta 34A, 469 (1978).

7. H. Frei, T.-K. Ha, R. Meyer and Hs. H. Günthard, Chem. Phys. 25, 271 (1977).

8. A. E. Shirk and J. S. Shirk, Chem. Phys. Lett. 97, 549 (1983).

9. W. F. Hoffman III and J. S. Shirk, Chem. Phys. 78, 331 (1983).

10. W. F. Hoffman III, A. Aspiala and J. S. Shirk, J. Phys. Chem. 90, 5706 (1986).

11. W. F. Hoffman III and J. S. Shirk, J. Mol. Struct., 157, 275 (1987).

12. A. K. Knudsen and G. C. Pimentel, J. Chem. Phys. 78, 6780 (1983).

13. P. Felder and Hs. H. Günthard, Chem. Phys. 85, 1 (1984).

14. J. Pourcin, G. Davidovics, H. Bodot, L. Abouaf-Marguin and B. Gauthier-Roy, Chem. Phys. Lett. 74, 147 (1980).

15. T. Lotta, J. Murto, M. Räsänen and A. Aspiala, J. Mol. Struct. 114, 333 (1984).

16. Hs. H. Günthard, J. Mol. Struct. 113, 141 (1984).

17. R. N. Perutz, Chem. Rev. 85, 97 (1985).

18. A. J. Barnes, J. Mol. Struct. 113, 161 (1984).

19. M. Poliakoff and J. J. Turner. In Chemical and Biochemical Applications of Lasers, ed. C. B. Moore, 5, 175 (1980).

20. S. H. Lin, X.-G. Zhang and G. Y. C. Wu, J. Chem. Phys. 85, 221 (1986).

21. J. Murto and M. Ovaska, Spectrochim. Acta A39, 149 (1983).

22. L. Fredin, B. Nelander and G. Ribbegard, J. Chem. Phys. 66, 4073 (1977).

23. L. Shriver, A. Burneau and J. P. Perchard, J. Chem. Phys. 77, 4926 (1982).

24. A. Aspiala, T. Lotta, J. Murto and M. Räsänen, Chem. Phys. Lett. 112, 469 (1984).

25. M. Räsänen, A. Aspiala, T. Lotta and J. Murto, Chem. Phys. Lett. 106, 102 (1984).

26. Hs. H. Günthard, J. Mol. Struct. 80, 87 (1982).

27. M. Räsänen and V. E. Bondybey, J. Phys. Chem. 90, 5038 (1986).

28. A. Aspiala, T. Lotta, J. Murto, M. Räsänen and V. P. Gupta, Chem. Phys. Lett. 109, 179 (1984).

29. T. Lotta, J. Murto, M. Räsänen, A. Aspiala and P. Särkkä, J. Chem. Phys. 82, 1363 (1985).

30. J. S. Binkley, M. J. Frisch, D. J. DeFrees, K. Raghavachari, A. Whiteside, H. B. Schlegel, E. M. Fluder and J. A. Pople, Department of Chemistry, CarnegieMellon University, Pittsburgh, PA.

31. J. Murto, M. Räsänen, A. Aspiala and T. Lotta, J. Mol. Struct. 108, 99 (1984).

32. J. Murto, M. Räsänen, A. Aspiala and T. Lotta, J. Mol. Struct. (Theochem.) 122, 213 (1985).

33. A. Aspiala, J. Murto and P. Stén, Chem. Phys. 106, 399 (1986).

34. M. Räsänen, J. Murto and V. E. Bondybey, J. Phys. Chem. 89, 3967 (1985).

35. J. Murto, M. Räsänen, A. Aspiala and L. Homanen, J. Mol. Struct. 92,45 (1983).

36. J. Pourcin, M. Monnier, P. Verlaque, G. Davidovics, R. Lauricella, C. Colonna and H. Bodot, J. Mol. Spectr. 109, 186 (1985).

37. M. Räsänen, A. Aspiala and J. Murto, J. Chem. Phys. 79, 107 (1983).

38. H. Kunttu, M. Dahlqvist, J. Murto and M. Räsänen, J. Phys. Chem. 92, 1495 (1988).

39. E. Hirota, J. Chem. Phys. 37, 283 (1962).

40. J. R. Durig, S. E. Godbey and J. F. Sullivan, J. Chem. Phys. 80, 5983 (1984). 
41. H. Kunttu, T. Raaska, J. Murto and M. Räsänen, J. Mol. Struct. (Theochem.) (1988).

42. J. R. Durig, S. E. Godbey and J. F. Sullivan, J. Chem. Phys. 80, 5983 (1984).

43. S. S. Butcher, R. A. Cohen and T. C. Rounds, J. Chem. Phys. 54, 4123 (1971).

44. P. Huber-Wälchli and Hs. H. Günthard, Spectrochim. . Acta 37A, 285 (1981).

45. M. Räsänen and V. E. Bondybey, Chem. Phys. Lett. 111, 515 (1984).

46. M. Räsänen and V. E. Bondybey, J. Chem. Phys. 82, 4718 (1985).

47. M. Räsänen, G. P. Schwartz and V. E. Bondybey, J. Chem. Phys. 84, 59 (1986).

48. W. Schultz and D. M. Kolb, J. Chem. Soc. Farad. Trans. II, 70, 1098 (1974).

49. A. Aspiala, T. Lotta, J. Murto and M. Räsänen, J. Chem. Phys. 79, 4183 (1983).

50. R. P. Müller, M. Nonella, P. Russegger and J. R. Huber, Chem. Phys. 87, 351 (1984).

51. O. Schrems, Ber. Bunsenges. Phys. Chem. 89, 297 (1985).

52. A. J. Barnes and R. M. Bentwood, J. Mol. Struct. 112, 31 (1984).

53. H. Takeuchi and M. Tasumi, Chem. Phys. 70, 275 (1982).

54. L. Homanen and J. Murto, Chem. Phys. Lett. 85, 322 (1982).

55. M. Räsänen et al. (in press).

56. M. Räsänen, A. Aspiala, L. Homanen and J. Murto, J. Mol. Struct. 96, 81 (1982).

57. H. Takeuchi and M. Tasumi, Chem. Phys. 77, 21 (1983).

58. M. Nakata and M. Tasumi, Spectrochim. Acta 41A, 341 (1985).

59. M. Nakata and M. Tasumi, Spectrochim. Acta 41A, 1015 (1985).

60. M. Nakata, M. Tasumi, Y. Hamada and M. Tsuboi, Chem. Lett. 467 (1983).

61. A. Shriver, S. Racine and L. Shriver, Chem. Phys. 100, 377 (1985).

62. S. Racine, A. Shriver, L. Shriver and L. P. Perchard, J. Mol. Struct. 118, 197 (1984).

63. H. Hollenstein, T.-K. Ha and Hs. H. Günthard, J. Mol. Struct. 146, 289 (1986).

64. T. Lotta, J. Murto, M. Räsänen and A. Aspiala, Chem. Phys. 86, 105 (1984).

65. J. Murto et al., unpublished results.

66. V. P. Gupta, T. Lotta, J. Murto, M. Räsänen and A. Aspiala, Chem. Phys. 90, 291 (1984).

67. T. Beech, R. Gunde, P. Felder and Hs. H. Günthard, Spectrochim. Acta 41A, 319 (1985).

68. H. Kunttu, J. Murto, T. Raaska and M. Räsänen (in press). 